\title{
Éditorial: Les instruments d'évaluation des pensionnaires et leur utilité pour la planification et la recherche en soins de santé
}

Le vieillissement démographique, particulièrement dans les pays industrialisés, impose un surcroît de demandes aux services de santé. Les personnes âgées, dont les problèmes de santé sont variés et complexes, consomment une large part des services de santé. Jumelée aux contraintes financières, la hausse du coût des soins de santé, attribuable en grande partie aux personnes âgées, a pris des proportions inquiétantes au Canada et dans d'autres pays. Les décideurs, les organismes de réglementation et les fournisseurs de services doivent relever le défi de réduire les coûts des soins de santé des personnes âgées tout en maintenant la qualité de ces soins.

Le U.S. Institute of Medicine définit la qualité des soins comme «le niveau des services de santé offert aux individus et aux populations qui augmentent les chances d'améliorer l'état de santé et qui correspondent aux connaissances actuelles de la profession» (traduction libre) (Lohr \& Walker, 1990). En 1983, le Congrès américain a demandé à l'Institute of Medicine d'examiner à l'échelle nationale le système des maisons de soins infirmiers, qui contribue pour une bonne part au coût des soins de santé, et de recommander des moyens de l'améliorer. L'une des principales recommandations de l'Institut porte sur la mise sur pied d'une évaluation uniforme et globale des pensionnaires. Les articles du présent numéro illustrent l'utilité du Resident Assessment Instrument (RAI), issu de cette recommandation, pour intervenir sur la qualité des soins aux personnes âgées.

Le RAI a été élaboré par la Health Care Financing Administration (HCFA), organisme fédéral chargé d'établir les normes sur les maisons de soins infirmiers. Le Congrès américain a donné à la HCFA le pouvoir de mettre sur pied le RAI et de réglementer son utilisation dans l'Omnibus Budget Reconciliation Act de 1987 (OBRA-87). Le nouvel instrument et le nouveau règlement de la HCFA sont en place depuis le printemps 1991 et s'appliquent maintenant à plus de 90 pour cent des maisons de soins infirmiers américaines.

La version du RAI à l'usage des maisons de soins infirmiers a été élaborée par une équipe multidisciplinaire de professionnels de la santé. Elle comprend trois composantes essentielles. La première de ces composantes est un ensemble de points d'évaluation qui donnent un profil global de chaque pensionnaire. Cette composante, appelée le Minimum 
Data Set (MDS), couvre des dimensions variés: les antécédents et les habitudes, la communication et l'audition, les problèmes physiques, fonctionnels et structurels, l'humeur et le comportement, les maladies diagnostiquées, l'état oral/nutritionnel, l'état de la peau, les procédures et traitements particuliers, la continence, la poursuite des activités, l'état de santé, l'état oral/dentaire et l'usage de médicaments. La deuxième composante du RAI est un ensemble de protocoles d'évaluation des pensionnaires (Resident Assessment Protocols ou RAPs) conçus pour jumeler les renseignements des MDS et les décisions portant sur les plans d'intervention. Les RAPs précisent les évaluations supplémentaires très ciblées à effectuer si le MDS d'un pensionnaire laisse voir des problèmes actuels ou potentiels. La troisième composante du RAI est un manuel contenant des directives sur la manière de compléter les MDS, des procédures d'évaluation des RAPs et des directives cliniques sur l'élaboration des plans d'intervention.

Les maisons de soins infirmiers américaines doivent maintenant utiliser le RAI pour évaluer l'état des pensionnaires au moment de leur admission, lors de tout changement important de leur état de santé ou au moins une fois l'an. Il est possible d'augmenter la fréquence des évaluations pour vérifier l'effet des soins et déterminer les ajustements requis. Certains États utilisent également les données recueillies au moyen du RAI pour déterminer les pensionnaires ayant des besoins de soins complexes ou un taux d'incapacité élevé dans les activités de la vie quotidienne (AVQ). Ces données servent ensuite à ajuster les prestations du programme Medicaid aux maisons de santé, au moyen d'un algorithme, le Resource Utilization Group ou système RUGS-III. Les données du RAI servent à plusieurs autres fins, dont l'élaboration d'indicateurs de la qualité (IQ) sur la base des points du MDS. Par exemple, les IQ du MDS peuvent fournir une estimation de l'occurrence chez les pensionnaires de phénomènes comme le déclin de l'état cognitif et d'autres incidents touchant la qualité de vie, notamment les chutes.

La communauté internationale des fournisseurs de soins de longue durée a également trouvé de multiples utilités au RAI. Des gériatres, des chercheurs universitaires et d'autres professionnels engagés dans l'élaboration et l'utilisation d'outils d'évaluation normalisés afin d'améliorer les soins aux personnes âgées ont formé un organisme international appelé interRAI. Le RAI des maisons de soins infirmiers est employé fréquemment dans plusieurs pays du monde dans des traductions adéquates, ce qui permet d'effectuer des comparaisons entre pays. En outre, les membres d'interRAI travaillent à adapter le RAI à divers contextes comme les soins à domicile, les soins actifs, la santé mentale et les soins post-actifs afin d'assurer le suivi du cheminement des clients dans le système des soins de longue durée.

L'utilité du RAI des maisons de soins infirmiers pour améliorer la qualité des soins aux pensionnaires âgés a été évaluée aux États-Unis sous 
les auspices de la HCFA. Cette évaluation prétest post-test a porté sur les changements dans les procédures de soins et les résultats observés chez les pensionnaires sur une base longitudinale. Elle a reposé sur un large échantillon aléatoire de maisons de soins infirmiers. Cette étude a permis d'observer plusieurs résultats positifs: augmentation de la quantité et de l'exactitude de l'information contenue dans le dossier médical des pension. naires, plus grande intégralité de la planification des soins, amélioration de plusieurs procédures de soins dont la participation des pensionnaires et de leur famille à la planification des soins, réduction du déclin dans certains domaines comme l'état physique et cognitif et réduction du taux d'hospitalisation des pensionnaires sans hausse de la mortalité (Phillips, Hawes, Morris, Mor, \& Fries, 1996). Des études démontrent que la majorité des administrateurs et des employés des maisons de soins infirmiers ont une attitude positive relativement aux effets du RAI sur la qualité des soins, bien que des employés soulignent qu'il alourdit leurs tâches administratives (Marek, Rantz, Fagin, \& Kreji, 1996). Certains établissements ont également eu de la difficulté à utiliser correctement le RAI, ce qui s'est traduit par des données inexactes (Sgadari et al., 1997). Dans l'ensemble cependant, le RAI apparaît comme un outil utile pour la planification des soins.

De plus en plus, on utilise aussi le RAI à des fins de recherche. Des experts examinent les bases de données du RAI afin de déterminer les facteurs de risque et de pronostic de divers états de santé et d'évaluer les effets des interventions cliniques chez les aînés dans tout le système des soins de longue durée. Les tenants de cette tendance soulignent les nombreux avantages des bases de données en comparaison avec la recherche plus ponctuelle (Black, 1997).

Les études ponctuelles connaissent plusieurs limites. Il est notamment difficile d'en généraliser les résultats, car on a tendance à réaliser ces études dans un contexte étroit, souvent atypique. Les échantillons de petite taille peuvent aussi limiter la généralisation si certains participants sont atypiques. Ces échantillons restreignent de plus l'efficacité statistique de la comparaison entre divers groupes de clients et de la stratification selon les caractéristiques des clients qui pourraient influer sur l'interprétation des résultats. La rapidité avec laquelle les changements se produisent dans le système des soins de longue durée peut signifier que les conclusions d'une étude ponctuelle fastidieuse à réaliser ne seront déjà plus pertinentes au moment où on les obtiendra. La recherche ponctuelle tend également à coûter cher, de sorte qu'on ne peut réaliser qu'un petit nombre d'études pour examiner un ensemble défini de facteurs de risque et de pronostic, ou d'interventions. En outre, les études ponctuelles comportent parfois un dédoublement de la collecte de données, ce qui alourdit considérablement le fardeau pour les clients participants, qui se plaignent alors d'être "sur-étudiés». Par ailleurs, des cliniciens qui travaillent sur le terrain sans participer directement à la collecte des données subissent une interruption 
de leurs activités normales sans avoir l'impression que la recherche leur appartient.

Les bases de données dérivées d'outils comme la série des RAI offrent une solution de rechange attrayante aux recherches ponctuelles, car elles évitent certains de ces écueils. Les bases de données communes à tout le système des soins de longue durée permettent la participation de plusieurs milieux, d'où des possibilités de généralisation accrues. On peut ainsi produire des échantillons de grande taille, ce qui accroît également les perspectives de généralisation et l'efficacité statistique. Comme l'information est recueillie régulièrement, la recherche peut s'effectuer rapidement et à moindre coût. On peut éviter le dédoublement des efforts de collecte et réduire ainsi le fardeau de la recherche sur la clientèle. Plusieurs études ponctuelles pourraient par exemple utiliser des renseignements sur l'état physique des clients provenant de la même base de données, ce qui éviterait à ces derniers de subir des mesures répétées. Les divers cliniciens qui recueillent normalement les renseignements de la base de données se sentiraient davantage au cœur de l'effort de recherche et le sentiment d'appartenance s'étendrait au-delà du petit nombre de cliniciens participant directement à des études particulières.

En dépit de ces avantages, la recherche sur base de données soulève des inquiétudes chez certains observateurs. La principale est qu'une mesure globale comme le RAI, conçue pour recueillir des données sur tous les aspects de l'état d'un client pertinents du point de vue des soins à apporter, a peu de chances de traiter ces aspects avec assez de profondeur pour répondre aux besoins de la recherche (Teresi \& Holmes, 1992). Les partisans de la base de données rétorquent que la pratique clinique et la recherche de qualité nécessitent toutes deux des données précises (Hawes, Phillips, Mor, Fries, \& Morris, 1992). L'exactitude n'est cependant pas le seul enjeu. La présence de renseignements sur des variables spécifiques nécessaires à la vérification d'une hypothèse et la nature des détails disponibles sont évidemment des points essentiels. Le manque de renseignements a déjà limité sérieusement la recherche sur base de données par le passé, situation qui risque de perdurer. Même s'il était possible, malgré les contraintes administratives, d'inclure dans une base de données toutes les variables qui pourraient devenir importantes pour la recherche future, il serait difficile de les prévoir d'avance. De même, les outils de mesure qui servent à recueillir les renseignements des bases de données risquent de devenir dépassés avec la création de nouveaux outils.

On s'inquiète aussi de l'exactitude des renseignements contenus dans les bases de données. Il faut normaliser les instruments de recherche dans la mesure du possible, mais certains aspects du MDS ne sont pas normalisés (Teresi \& Holmes, 1992). Par exemple, le MDS et son manuel de formation ne précisent pas d'ensemble standard d'éléments sur lesquels recueillir des données; le manuel illustre plutôt comment transformer la définition des éléments en questions d'interview. Par conséquent, chaque 
évaluateur peut décider le nombre d'éléments, le choix des éléments et la formulation à employer pour évaluer les pensionnaires. En outre, il peut y avoir des variations importantes selon le type de personnel qui administre le MDS, les sources consultées (pensionnaires, famille, autres membres du personnel) et le temps consacré à l'exécution de cette tâche. La variation de la mesure peut avoir des répercussions importantes sur la validité des données, particulièrement pour des concepts subjectifs comme le comportement. La fiabilité des données peut également être touchée par la variation entre les méthodes de collecte de données. Toutefois, on peut en dire autant de certains outils employés dans les études ponctuelles; ce n'est donc pas un problème exclusif à la recherche sur base de données.

Malgré les objections soulevées à propos des propriétés psychométriques des renseignements sur base de données (Ouslander, 1994), objections qui peuvent d'ailleurs s'appliquer aussi aux outils employés dans les études ponctuelles, de plus en plus d'observations laissent entendre que les données du RAI sont fiables au plan psychométrique. Tous les instruments du RAI ont été élaborés dans un processus généralisé de consultation et d'examen, processus qui contribue à la validité apparente et de contenu. On a examiné la validité concourante en déterminant si les domaines de l'instrument, le diagnostic d'une maladie et l'usage de médicaments, par exemple, sont corrélés logiquement (Gambassi et al., 1998). On a également réalisé un certain nombre d'études concurrentes sur la validité; il a par exemple été démontré que l'échelle de rendement cognitif du MDS a un taux de corrélation d'environ 0,80 avec le mini-examen de l'état mental (Morris et al., 1994). De plus, on a constaté la validité prédictive du MDS; une étude démontre notamment que les données des MDS sur les chutes chez les pensionnaires de maisons de soins infirmiers sont de bons prédicteurs de chutes subséquentes (Lipstiz et al., 1994).

Depuis quelques années, le RAI fait également l'objet d'études de fiabilité. Le coefficient d'objectivité des éléments du MDS, tel qu'établi aux États-Unis, varie de 0,40 pour des concepts subjectifs comme l'humeur et le comportement à 0,70 ou plus pour des concepts plus objectifs comme les aptitudes cognitives et décisionnelles ou le rendement personnel dans les AVQ (Morris et al., 1997). En comparaison avec les résultats de recherche, ces coefficients d'objectivité sont semblables dans toutes les régions des États-Unis (Sgadari et al., 1997), tout comme le sont les dossiers cliniques tenus à jour régulièrement (Phillips \& Morris, 1998). L'indice d'engagement social, une des rares mesures sommaires du MDS, montre un niveau élevé de cohérence interne (Mor et al., 1995). Ni la constance test-retest du MDS, ni la sensibilité de cet outil à la détection de changements, un concept relié au précédent, n'ont fait l'objet d'études précises, bien que des études de résultats aient permis de constater des changements dans l'état des pensionnaires.

Les études comparatives nationales ou internationales qui utilisent les données du MDS soulèvent d'autres inquiétudes, dont certaines s'ap- 
pliquent encore, à un degré variable, à la recherche ponctuelle. Dans les études comparatives entre des sites d'un même pays ou de pays différents, l'ajustement du risque sur la base de l'état du client devient une considération importante (Berlowitz et al., 1996). Il serait inopportun, par exemple, de comparer les taux de chute dans des maisons de soins infirmiers situées dans des cadres différents sans ajuster le risque de chute de leurs pensionnaires sur la base de facteurs comme l'état physique. De même, il faut tenir compte du biais potentiel dans les réponses de sujets de cultures différentes, notamment dans le signalement de la douleur. Les employés peuvent également introduire différents biais dans les réponses, ce phénomène risque d'être plus présent lorsque des données utilisées à des fins de recherche servent à la planification ou au remboursement des soins. Les employés pourraient noter que des pensionnaires ont maintenu ou amélioré leur état afin d'éviter de déclencher des évaluations plus longues ou afin de donner une image de réussite. À l'inverse, ils pourraient aussi noter que les pensionnaires déclinent afin de se soustraire à un programme impopulaire ou de maximiser le financement. De tels facteurs compliquent non seulement les études entre établissements d'un même pays ou de pays différents, mais aussi les études longitudinales à l'intérieur d'un même établissement, où les biais de déclaration peuvent varier avec le temps.

Il n'en reste pas moins que des bases de données soigneusement élaborées et compilées à partir d'instruments comme le RAI offrent d'excellentes perspectives de recherche valables pour la société. Les données du RAI comme l'illustre le présent numéro, peuvent servir à dresser le profil de groupes de clients, à explorer les associations entre les variables des clients et à mener des essais cliniques non randomisés. Dans ce numéro, Phillips et Spry comparent les pensionnaires de maisons de soins infirmiers américaines ayant des antécédents de maladie mentale chronique et les autres pensionnaires, au plan des caractéristiques démographiques, de l'état fonctionnel, des comportements et des interventions thérapeutiques; de leur côté, Hirdes et ses collègues font une description transnationale des pensionnaires de maisons de soins infirmiers chez qui on a diagnostiqué une dépression et l'usage d'antidépresseurs au Canada, au Japon, en Islande et en République tchèque. Finne-Soveri et ses collègues examinent le diagnostic des maladies, l'incapacité physique, l'incapacité cognitive et le sexe en tant que prédicteurs possibles de la douleur chez les pensionnaires de maisons de soins infirmiers dans les pays nordiques; pour leur part, Gilbart et Hirdes évaluent la santé, le stress, l'engagement social et le soutien social en tant que prédicteurs possibles du bien-être psychosocial chez les pensionnaires d'établissements canadiens. Gambassi et ses collègues incorporent les données du MDS à une évaluation des effets de la digoxine par opposition aux inhibiteurs de l'enzyme de conversion et au traitement combiné sur le taux de déclin des AVQ chez les pensionnaires de maisons de soins infirmiers atteints d'insuffisance cardiaque globale aux États-Unis. Enfin, Morris et ses collègues 
et Björkgren et ses collègues traitent respectivement de la fiabilité transnationale du RAI des soins à domicile au Canada, aux États-Unis, au Japon et en Italie et de la validation d'une version du système de classification de la clientèle RUGS-III adaptée aux soins à domicile aux ÉtatsUnis. Tous ces auteurs ont pris soin de traiter des problèmes potentiels reliés à la recherche sur base de données dans leurs propres études.

Certains articles de ce numéro comprennent des données canadiennes. Au Canada, l'utilisation du RAI a été autorisée dans les unités de soins de longue durée en Ontario et dans tous les établissements de soins de longue durée de la Saskatchewan. Des études d'évaluation du RAI sont en cours dans d'autres provinces, dont l'Alberta. L'Alberta a récemment mis au point un nouvel outil qui incorpore des éléments du MDS, soit un instrument de détermination des besoins en soins de longue durée (Continuing Care Needs Determination Instrument ou CCNDI). Ainsi, de plus en plus de bases de données du RAI sont à la disposition des chercheurs canadiens intéressés à l'étude des soins de santé aux personnes âgées.

Les avantages des bases de données de grande qualité pour la planification et la recherche en matière de soins de santé sont reconnus depuis plusieurs années. Le RAIMDS représente un jalon important dans l'établissement progressif d'un langage d'évaluation commun à toutes les professions et à tous les cadres de soins. Les chercheurs travaillent avec des bases de données doivent notamment s'assurer de compléter l'information disponible pour que des questions spécifiques puissent être étudiẹes, de mesurer la fiabilité des données spécifiques et de tenir compte de facteurs comme l'ajustement du risque et les biais dans les réponses. Les développements de la technologie informatique concrétisent la possibilité d'établir des bases de données longitudinales valables en matière de soins de santé. Les cliniciens et les chercheurs peuvent accroître la valeur de ces bases de données en travaillant ensemble à en optimiser la conception et la gestion.

\section{Références}

Berlowitz, D.R., Ash, A.S., Brandeis, G.H., Brand, H.K., Halpern, J.L., \& Moskowitz, M.A. (1996). Rating long term care facilities on pressure ulcer development: Importance of case-mix adjustment. Annals of Internal Medicine, $124,557-563$.

Black, N. (1997). Developing high quality clinical databases. British Medical Journal, 315, 381-382.

Gambassi, G., Landi, F., Peng, L., Brostrup-Jensen, C., Calore, K., Hiris, J., Lipsitz, L., Mor, V., \& Bernabei, R. (1998). Validity of diagnostic and drug data on standardized nursing home resident assessments: Potential for geriatric pharmaco-epidemiology. Medical Care, 36, 22-34.

Hawes, C., Phillips, C.D., Mor, V., Fries, B.E., \& Morris, J.N. (1992). MDS Data should be used for research. The Gerontologist, 32, 563-564.

Lipstiz, L., Nakajima, I., Gagnon, M., Hirayama, T., Conelly, C.M., \& Izumo, H. (1994). Muscle strength and fall rates among residents of Japanese and 
American nursing homes: An international cross-cultural study. Journal of the American Geriatrics Society, 42(9), 953-959.

Lohr, K.N., \& Walker, A.J. (1990). The utilization and quality of control peer review organization. Dans Medicare: A strategy for quality assurance. Washington, DC: Institute of Medicine/National Academy Press.

Marek, K.D., Rantz, M.J., Fagin, C.M., \& Kreji, J.W. (1996). OBRA-87: Has it resulted in better quality of care? Journal of Gerontological Nursing, October, 28-36.

Mor, V., Branco, K., Fleishman, J., Hawes, C., Phillips, C., Morris, J., \& Fries, B. (1995). The structure of social engagement among nursing home residents. Journals of Gerontology: Psychological Sciences, 50, 1-8.

Morris, J.N., Fries, B.E., Mehr, D.R., Hawes, C., Phillips, C., Mor, V., \& Lipsitz, L. (1994). MDS cognitive performance scale. Journal of Gerontology: Medical Sciences, 49(4), M174-M182.

Morris, J.N., Nonemaker, S., Murphy, K., Hawes, C., Fries, B.E., Mor, V., \& Phillips, C.D. (1997). A commitment to change: Revision of HCFA s RAI. Journal of American Geriatrics Society, 45, 1011-1016.

Ouslander, J.G. (1994). Maximizing the Minimum Data Set. Journal of the American Geriatrics Society, 42, 1212-1213.

Phillips, C.D., Hawes, C., Morris, J., Mor, V., \& Fries, B.E. (1996). Effects of the RAI on quality of care: Executive summary. Research Triangle Park, NC: Research Triangle Institute, Program on Aging and Long-Term Care.

Phillips, C.D., \& Morris, J.P (1998). The potential for using clinical and administrative data to analyze outcomes for the cognitively impaired: An Assessment of the Minimum Data Set for Nursing Homes. Alzheimer Disease and Associated Disorders, 11, 162-167.

Sgadari, A., Morris, J.N., Fries, B.E., Ljunggren, G., Jonsson, P.V., DuPaquier, J.-N., \& Schroll, M. (1997). Efforts to establish the reliability of the RAI. Age and Ageing, 26(suppl. 2), 27-30.

Teresi, J.A., \& Holmes, D. (1992). Should MDS data be used for research? The Gerontologist, 32, 148-149. 